\section{LES SOURCES D'ÉNERGIE PEU CONNUES}

\section{LA TOURBE}

(Suite et Fin)

\section{COMBUSTION DANS LES FOYERS INDUSTRIELS}

Grilles a Échelons. - Les grilles à échelons sont les plus efficaces pour la bonne utilisation des combustibles pauvres.

Nous n'entreprendrons pas la description, d'ailleurs fort simple, d'une grille à échelons ordinaires. Nous nous bornerons à mentionner les résultats que ces foyers ont permis d'obtenir avec la tourbe, pour le chauffage des générateurs de vapeur : A Granefors (Suède), I kg de lourbe donne / kgs de vapeur et à Lristbrau (Allemagne), $\{, 5 \mathrm{kgs}$.

Foyers Godnlot. - La Maison Godulot de Paris construit un foyer à échelons avec alimentation automatique pour combustibles pauvres et qui s'adapte particulièrement bien à la tourbe, surtout lorsque la tencur en cendres est faible el que le séchage péut en être effectué dans de bonnes conditions. Ce foyer est représenlé par la figure I

La grille se compose de barreaúx semi-cinculaires à diamètre progressif et se recouvrant comme des lames de persiennes, le foyer ayant ainsi une forme troncônique. L'alimentation en combustible est assurée automatiquement par un transporteur à hélice à auget croissant, cette dernière disposition ayant pour but de prévenir les rentrées d'air accidentelles occasionnées par des chargcments fréquents.

Autres foyers a ÉGHelons. - A l'usine d'Oriechovo on brûle la tourbe s'ur une grille mécanique à échelons Kowalsky (I). Un barreau sur deux est mobile de façon à assurer l'écoulement du icomb'ustible et de la cendre vers la partie inférieure de la grille. La consommation de combustible est de I $\mathrm{kg}$. de tourbe pour une production de 4. roo $\mathrm{kgs}$ de vapeur à I5 atmosphères.
La salle des chaudières comprend quatre chaudières à tubes d'eau et surchauffeurs alimentées par un épurateur et par l'eau de condensation des turbines. L'eau de circulation pour la condensation, fournie par le marais, contenant une forte proportion d'acides organiques, toutes les pièces métalliques exposées au contact de cette eau sont en fonte ou en bronze. Les tubes de laiton du condenseur à surface sont constitués par un alliage à haute teneur en bronze et étamés. Ces mesures de précaution ont donné de bons résultats.

Les foyers sont constitués par des grilles à échelons dont l'inclinaison sur l'horizontale est de 36 degrés. Chaque grille est en deux parties ayant chacune une surface de $4 \mathrm{~m}^{2}+\mathrm{c}$ alimentées alternativement par une même trémie. Les chargements ayant lieu fréquemment par suite du faible pouvoir calorifique du combustible (2.50o à $3.500 \mathrm{cal}$, par $\mathrm{kg}$.) et do son grand volume spécifique (roo $\mathrm{kg}$. de briquettes occupent un volume de $0.4 \mathrm{~m}^{3}$ ), il convenait d'éviter les entrées d'air' accidientelles. Ce but a été alleint au moyen de la tourbe elle-même, qui tombant rapidement des trémies, forme oblurateur automatique.

De nombreuses difficultés ont été rencontrées au début,par suite de la variabilité d'u degré d'humidité du combustible, de mélange de tourbe de surface ou de sable, du gel de la tourbe pendant l'hiver, etc Actuellement, toutes ces difficultés ont été surmontées.

La consommation de combustible est de 2,4 à $2,8 \mathrm{kgs}$ par kw.-h., ce qui porte la consommation annuelle de la centrale à 25.000 tonnes de briquettes. De vastes hangars, situés ì proximité, emmagasinent une réserve pouvant suffire pour une année. Les frais de premier établissement de l'installation montent à 4 à 5 millions de francs.

Grilles planes. - Les grilles planes pour la combustion de la tourbe devront avoir un écartement entre barreaux

RÉSULTATS DES ES6AIS COMPARATIFS DES DIVERS COMBUSTIBLES SUR GRILLE PLANE

\begin{tabular}{|c|c|c|c|c|c|c|c|}
\hline COMBUSTIBLE & $\begin{array}{c}\text { DURÉE } \\
\text { DE L'ESSAI } \\
\text { HEURES }\end{array}$ & $\begin{array}{l}\text { COMBUSTIBLE } \\
\text { PAR HEURE } \\
\text { ET M } \\
\text { DE SURFACE } \\
\text { DE GRILLE } \\
\text { KILOGS }\end{array}$ & $\begin{array}{l}\text { TEMPÉRATURE } \\
\text { DE L'EAU } \\
\text { d'alimentation } \\
\text { DEG. CENT. }\end{array}$ & $\begin{array}{l}\text { VAPORISATION } \\
\text { HORAIRE PAR } \\
M^{2} \text { DE SURFACE } \\
\text { DE GRILLE } \\
\text { KILUGS }\end{array}$ & $\begin{array}{l}\text { EVAPORATION } \\
\text { PAR KILOG. } \\
\text { DE } \\
\text { COMBUSTIBLE } \\
\text { KILOGS }\end{array}$ & \multicolumn{2}{|c|}{$\begin{array}{l}\text { POILS DE TOURBE ÉQUIVALENT } \\
\begin{array}{l}\text { A } 1 \text { KILOG DE HOUILLE } \\
\text { SIMPLE } \\
\text { KILOGS }\end{array} \begin{array}{c}\begin{array}{c}\text { MÉLANGÉS DE } \\
\text { KIO DE TOURBE } \\
\text { KILOGS }\end{array}\end{array}\end{array}$} \\
\hline Houille Suédoise (Bille sholm) ........... & 7 & 0.9 & 83 & 6.4 & 7.00 & 1.38 & 1.21 \\
\hline Houille Anglaise (S. York).... . . . . . & 168 & 1.0 & 75 & 66 & 9.40 & 1.84 & 1.62 \\
\hline Houille Anglasse (N. Wales) . . . . . & 168 & 1.2 & 85 & 10.4 & 8.40 & 1.65 & 1.45 \\
\hline Tourbe de Lobevod ....... . . . . & 7 & 1.5 & 85 & 12.7 & 5.10 & - & - \\
\hline Houille N. Willes mélangés à $50 \%$ de tourbe. & 6 & 1.8 & 85 & 13.0 & 7.10 & - & - \\
\hline
\end{tabular}

\section{La Centrale de Wiesmoor}

Cette centrale à vapeur chauffée à la tourbe et exploitée par la Société Siemens Schuckert est installée ten plein centrc des tourbières de Wiesmoor qui l'alimentent en combustible. La superficie de ces marais est de plus de 6.000 hectares, l'épaisseur de la couche combustible étant,en moyenne de 3 mètres.

La puissance tolale de 5.400 chevaux est produite par trois turbo-alternateurs : deux groupes triphasés de $x_{250} \mathrm{~K}$. V. A. à 5000 V. tournant à 3000 T. M. el un groupe de 1550 K. V. A. à la même tension et à la même vitesse.

(1) On trouvera le plan de cette grille dans le rapport de NYsThov déjà mentionné. plus faible, la tourbc nécessitant moins d'air que le charbon. Le tableau ci-desisus donne le résultat des essais comparatifs effectués à la Fabrique de Sucre d'Oresund (Suède), d'après MM. Larson et Wallgren. Ces résultats montrent qu'on obtient 'un meilleur rendement en employant un mélange de tourbe et de houille à parties égales.

Grilles automatiques BABcock et Wrlcox. - Des essais ont été effectués récemment à la Poudrerie Nationale du Pont-de-Buis (Finistère) avec des foyers soufflés à chargement et décrassage automatiques. Le combustible était une tourbe provenant des tourbières voisines et contenant jusqu'à 40 pour roo d'eau. Un simple drainage pourrait permettre d'arriver à ce degré d'humidité et par suite, il serait 
possible d'utiliser directement la tourbe brute dans les foyers en supprimant les opérations du séchage.

La couche de tourbe chargée sur les grilles doit être assez épaisse, ce combustible brûlant plus vite que le charbon ; la vitesse de rotation des grilles doit rigalcment être augmentée. Pour une même vaporisation il y a lieu de compter sur un poids de tourbe double du poids de charbon de bonne qualité à 7.500 calories. On arrive, sans aucune difficulté à marcher à des allures horaires de 15 à $20 \mathrm{kgs}$ de vapeur au mètre carré de surface de grille. Les constructeurs espérent même dépasser sensiblement cette allure de combustion, de façon à obtenir une vaporisation horaire aussi importante qu'avec du charbon, soit de 18 à $25 \mathrm{kgs}$ par mètre carré de surface de grille.

Les essais, qui ont porté également sur la manutention mécanique du combustible, ont permis de constater que toute station centrale équipée pour le fonctionnement au charbon n'a pas, pour utiliser la tourbe sur les grilles soufflées BABCOCK ÈT WrLcox, à modifier les appareils existants, ce qui est un avantage appréciable.

\section{LA POUDRE DE TOURBE}

La tourbe, à l'état de poudre, n'absorbe plus l'humidilé ot possède une densité presque aussi élevée que celle du charbon. En outre, la combustion s'effectue complètement, en raison du mélange intime de l'air, et ne dégage presque pas de fumée.

La poudre de houille a été longtemps employée dans les fabriques de ciment, mais son prix de revient est très élevé, car il est nécessaire qu'elle soit très finement pulvérisée.

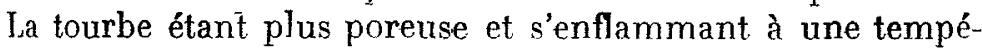
rature plus basse ne nécessite pas un grain aussi fin. Le combustible en poudre s'allume très facilement dans le foyer sans quie, cependant, il $\mathrm{y}$ ait danger d'explo-

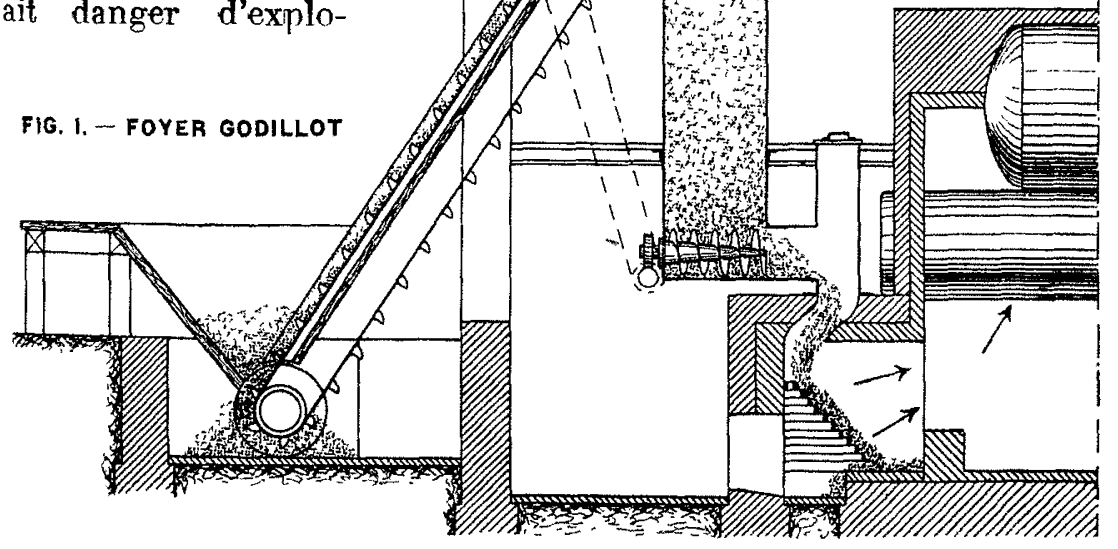

donne le produit fini, lequel, après un dernier passage dans un moulin, est mis en sacs.

La production des ateliers de BAEck (Suède) est de 15 tonnes de poudre à $x_{2} \%$ d'humidité par $2 /$ heures en partant de tourbe à $50 \%$ d'eau et de 2 I tonnes aver de la tourbe à $40 \%$ d'humidité. Le séchage est obtenu au moyen d'un four à poudre de tourbe consonmant respectivement 12 et 9 pour roo de la production suivant la teneur en humidité.

Le pnix de revient, tous frais compris, est de $12 \mathrm{fr} .7^{5}$ la tonne et le prix d'une installation produisant 12.000 tonnes de poudre par an est de 50.000 francs

Chaufrage a la Poupre de Tol nie. - Des essais effeclués à Stockholm au sujet du chauffage des chaudières à la poudre de tourbe ont donné les résultats suivants :

I kg. de poudre de tourbe à $7 \%$ d'humidité a donné une vaporisation de 5 k. 270 .

I kg. de houille de Newcastle a ćraporé dans les mêmes conditions $5 \mathrm{k}$. 700 .

Vaporisation horaire par mètre carré de surface de grille :

$$
\begin{aligned}
& \text { Poudre de tourbe......... } 18 \mathrm{kgs} . \\
& \text { Houille de Newcastle....... } 12 \mathrm{kgs} .
\end{aligned}
$$

On a imaginé plusieurs appareils spéciaux pour brûler la poudre de tourbe. Ie plus conmu est le foyer de Camp. II cst constitué par une trémie dans laquelle est chargée la poudre de tourbe. Celle-ci est puisée à l'extrémité inférieure de la trémie par une vis sans fin qui la déverso dans un tamis rotatif où elle est finement divisće et mise en suspension dans l'air primaire appelé par un ventilateur et qui l'entraîne dans le fover où s'effectuc la combustion.

I. a poudre de tourbe trouve également un emploj intéres- sion, ce qui est généralement le cas avec les combustibles pulvérisés ; il permet d'obtenir une température très élevée et facilement réglable, en flamme réductrice ou oxydante à volonté.

Fabrication de la Poudre de Tourbe (Procédé Ekelund). - La tourbe séchée à l'air et gelée passe d'abord dans un broyeur de dégrossissage constitué par deux cylindres cannelés tournant en sens inverse ; à la sortie de cet apparei] la poudre grossière tombe dans un finisseur à disques qui la pulvérise finement. Après criblage, elle est transportée au four de séchage, puis de nouveau sur un crible qui

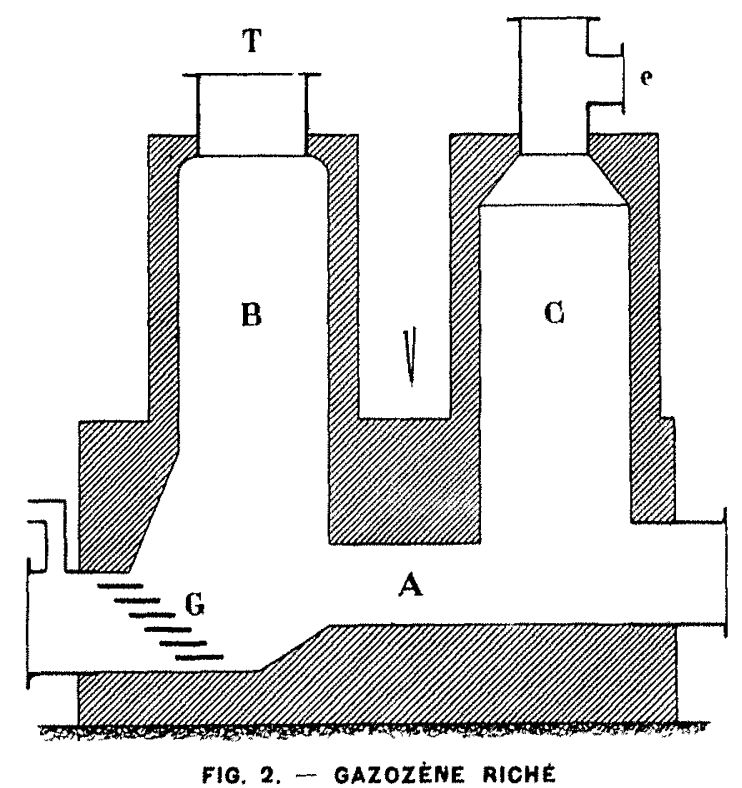

sant dans le chauffage des locomotives. Des essais ont étr effectués récemment en Suissc. Ils auraient, paraît-il, donné des résultats satisfaisants.

En Suède également, des essais ont été faits dans ce sens sous la direction de M. von Porat ; ces essais ont élé suivis avec intérêt par des ingénieurs français notamment.

La tourbe étant plus volumineusc que la houille nécessito un plus grand récipient pour l'emmagasinage. Ce récipient placé sur le tender est disposé de façon à produire un chargement automatique du foyer. La mise à feu s'effectue au moyen de charbon ou d'huile et la tourbe passe dans un appareil de Camp d'un type analogue à celui décrit ci-dessus 
Enrichussement de la Tourbe. - La tourbe étant un combustible à faible pouvoir calorifique mais étant, d'autre part, doué de qualités absorbantes très marquées, l'idée est venue de bonne heure d'en élever la valeur calorifique par absorplion de liquides riches en matières combustibles ; particulièrement des huiles et des pétroles.

Un calcul simple permet d'établir le pouvoir calorifique d'une tourbe que l'on aura imprégnée dans une certaine proportion, d'un hydrocarbure liquide à pouvoir calorifique élevé, 12.000 calories par exemple.

Dans le procédé Teissier, le seul pratiqué actuellement eu France à notre connaissance, la tourbe desséchée à l'air est mise en tas, arrosée d'huiles lourdes el pelletée comme du mortier. Le combustible obtenu en partant d'une tourbe à 3.000 calories a un pouvoir calorifique de plus de 7.000 calories ; il brûle avec une flamme très active et ne dégage que peu de fumće.

Des essais effectues sur une machine demi-fixe du type Wolff de $35 / 45$ chevaux ont donné les résultats suivants Cetle machine nécessitait une mise en marche de deux heures pour atteindre une pression de $\mathrm{r}_{2} \mathrm{kgT}$ avec chauffage au charbon tout venant. Avec la tourbe desséchée à l'air simplement, cette durée élait portée à quatre heures. Enfin, avec la tourbe enrichie elle était réduite à une heure dix minules.

\section{UTILISATION DANS LES GAZOGENES}

C'est là l'emploi de la lourbe paraissant le plus avantagcux. Oulre les avantages généraux de tous les gazogènes faible consommation de combustible, absence de fumées et de cheminées, simplicité du matériel et sécurité de la marche, les gazogènes à tourbe présentent celui de la possibilité de récupérer, dans les gaz engendrés, des prod'uits chimiques de grande valeur, el toul particulièrement le sulfate d'ammoniaque, qui abaissent le prix de revient de la forec inotrice dans une sensible proportion.

L'utilisation de la tourbe dans les gazogènes a nécessité l'étude d'appareils spéciaux, d'abord en raison de la consislance du combustible et, ensuite, en raison de la quantité importante de produits bitumineux condensables en suspension dans les gaz. Des épurateurs doivent êíre prëvus pour l'élimination de ces goudrons avanl l'arrivée des gaz aux appareils d'utilisation : moteurs ou brûleurs, où ils se déposeraient et, par carbonisation, donneraient un dépôt adhérent qui entraînerait bientôt un arrêt du fonctionnement. Ie principe de ces appareils pour combustibles bitumineux a été indiqué, pour la première fois, par l'ingénieur anglais MoNd.

Plusieurs installations fontionnent d'après ce procédé en Angleterre, en Allemagne et particulièrement en Italie aux Usines de Codisoro et d'Orentaro de la Società per l'utilizzazione dei Combustibili Italiani.

Nous passcrons d'abord en revue les divers générateurs que l'on trouve actuellement sur le marché, anrès quoi nous examincrons les résultats obtenus dans diverses installations cxistantes.

Gazonène Riché. - La Maison Riché de Paris construit un gazogène pour combustihles tels que les sciures et déchets de bois qui admet également l'emploi de la tourbe.

Je gazogène Riché est du type à deux combustibles et à deux cuves, celles-ci étant comme d'ordinaire, constituées par une maçonnerie en matériaux réfractaires à revêtements métalliques et réunies entre elles, à leur partie inférieure par vne conduite A (fig. 2). La tourbe est chargée par la trémie $\mathrm{T}$ et brûle sur une grille à gradins $\mathrm{G}$ par laquella l'air primaire est injecté. La vapeur d'eau est fournie par le cendrier. Les produits de la distillation qui parcourent donc la cuve $B$ de haut en bas arrivent ensuite à la partie inférieure de la cuve $\mathrm{C}$ remplie de coke ou de charbon de bois et s'échappent finalement par la canalisation $e$.

Gazogène Guardabassi Goulliard, - Cet appareil conçu dès avant la guerre par les inventeurs, est basé sur un principe nouveau permettant la récupération directe des sousproduits au fur et à mesure de leur formation, par prise directe des gaz dans la colonne de distillation aux différentes ha'uteurs. On peut le comprendre sans figures.

Le gazogène se compose d'une maçonnerie réfractaire à revêtèment métallique à l'inléricur de laquelle se trouve placée verticalement la cornue de distillation du combustible. Celle-ci se compose de quatre éléments en fonte, indépendants et pourvus, à leur partic supérieure, sauf le premier dans lequel s'effectue le séchage de la tourbe, d'une tubulure de prise des gaz.

Le chargement de l'appdreil s'effeclue d'une façon continue au moyen d'un élévaleur à godets déchargeant la tourbe dans une trémie d'alimentation à double obturation, cela afin d'éviter les rentrées d'air qui nuiraient à la bonne marche de la distillation.

La lourbe descendant le long de la cornue est brasséc automaliquement par suite de la forme même des éléments et se Irouve soumise à des températures constamment croissantes et atteignant $\mathbf{r}$.ooo degrés à la partie inférieure. A ce moment, elle se trouve alors complètement transformée en coke qui s'éteint dans une cuve à eau d'où une vis sans fin animée d'un mouvement ininterrompu en assure l'extraction d'une façon continue.

En outre, l'extinction du çke prodvit un dégagensent de vapeur qui se mélange à l'air primaire réchauffé par son passage dans le récupérateur de chaleur. Celui-ci est constitué par quatre chambres radiales travaillant deux à deux alternativement à la récupération et au réchauffage.

Le foyer, conılèbonent indépendant du'massif du form est constitué par : une grille ordinaire inclinée à ıo degrés ; une chambre de nettoyage qui permet un décrassage facile ef un contrôle constant ; un cendrier de grande capacité et un réservoir à combustibles continuellement rempli qui alimente le foyer. L'inclinaison des parois de ce dernier yermet au combustible de glisser aisément jusqu'à la grille el dy former une couche d'épaisseur constante.

Voici les résultats obtenus avec un four de ce modèle établi pour traiter 30 tonnes de tourbe à 40 pour roo d'humidité par 24 heures, quantité correspondant environ à 12 tonnes de tourbe sèche.

Gaz incondensables (utilisables pour la production de la force motrice on le chauffage)

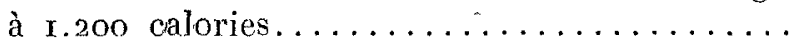

Huile pyrogénée distill ${ }^{t}$ au-dessous de $270^{\circ} \mathrm{c}$.

Huile pyrogénée distill ${ }^{t}$ au-dessus de $270^{\circ} \mathrm{c}$.

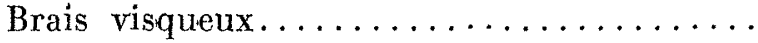

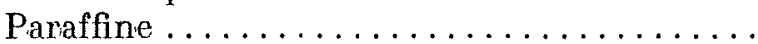

Sulfate d'ammoniaque (tourbe à ' $.5 \%$ d'azote)

Acétate de chaux à 80 pour roo..........

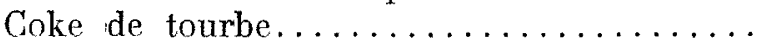

$2.400 \mathrm{mc}$
$240 \mathrm{kil}$
$360 "$
$240 "$
$240 "$
$405 "$
$240 "$
$7.800 "$

Gazogène Winterthur. - La Société suisse pour la construction de locomotives et de machines à Winterthur, vient de créer un nouveau type d'installation de gazogène à tourbe 
à récupération des sous-produits dont les essais ont eu lieu dernièrcment.

L'installation comporte un gazogène du lype ordinaire, mais dont les formes et les dimensions ont été adaptées à la nature spéciale de la tourbe. C'est ainsi que, par exemple, la hauteur de la couche du combustible a été augmentée ; cette disposition permet l'emploi de tourbe ayant une teneur en humidité atteignant 5o pour roo. Le combustible restant plus longtemps dans le générateur est séché par les gaz à haute température dont une partie de la chaleur est ainsi récupérée. A leur sortie du gazogène, ceux-ci traversent un condenseur cylindrique dans lequel ils se refroidissent tt abandonnent une partie de leur humidité.

A la suite de ce condenseur vient un épurateur centrifuge à injection d'eau composé d'une série de roues à aubes mélangeant le gaz et l'eau pulvérisée injectée. Cet appareil a pour but de débarrasser le gaz de ses goudrons, ceux-ci étant entraînés par l'eau dans une cuve où ils sont récupérés.

Les gaz épurés sont aspirés par la dernière aube, agissant comme ventilatelur ct sont chassés dans un scrubber à cokc humide où ils subissent un dernier lavage et refroidissement. Avant d'être envoyés au moteur, ils peuvent encore au besoin traverser un filtre qui retient les dernières parcelles de goudron, mais l'adjonclion de cet appareil n'est pas toujours nécessaire.

Les essais faits à Winterthur avec un gazogène de roo chevaux alimentant un moteur de 60 chevaux et un moteur de 40 chevaux ont donné les rés'ultats suivants :

Le combustible employé était une tourbe fibreuse d'un pouvoir calorifique de $2.200 \mathrm{cal}$. contenant 50 pour roo d'humidité et 2 à 3 pour roo de cendres, sous forme de briquettes mesurant $35 \mathrm{~cm} . \times$ ro $\mathrm{cm} . \times 8 \mathrm{~cm}$. Lianalyse chimique du gaz a donné la composition suivante :

Acide carbonique de........ 7.8 à 8.5 pour 100

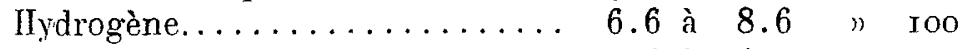

Méthane.............. 3.4 à 4.5 \# I0o

Oxyide de carbone.......... 20 à 21.8 " 100

Le pouvoir calorifique variant de $x .050$ à $x$. I 80 calories au mètre cube.

La consommation de combustible a été de r,415 $\mathrm{kgs}$ par cheval-heure effectif, soit 3. I 5 cal. par cheval-heure effectif, ce qui correspond à un rendement thermique supérieur à 20 pour roo.

GAZOGÈne LYMN. - L'air préalablement chargé de vapeur d'eau et réchauffé dans un appareil de récupération calorifique des gaz est soufflé sous la grille du gazogène. Il traverse la masse du combustible qui distille à une température suffisamment basse pour que les goudrons ne soient pas décomposés et sort par la partie supérieure. Après échange calorifique dans un réchauffeur de l'air primaire les gaz arrivent dans une chambre où ils sont mis en contact avec une solution pulvérisée de sulfate d'ammoniaque légèrement acide. II a été reconnu que cette solution constituait le meilleur mode de fixation de l'ammoniaque.

Les gaz débarrassés des produits ammoniacaux passent dans un refroidisseur de construction analogue à l'annareil précédent, mais où la solution est remplacée par de l'eau froide. Cet apnareil fonctionne, en circuit avec le saturateur d'air primaire.

L'appareil de récupération de l'ammoniaque et le refroidisseur sont basés sur l'inertie des gaz. Ils se composent d'un cvlindre vertical dans lervel sont disposées le long de l'axe. des cuvettes fronc-cônioues, portant une ouverture circulaire au centre. L'axe porte des plateaux, en nombre égal à celui des cuvettes, légèrement côniques et animés d'un mouvement de rotalion. Suivant l'axe arrive un tube qui déverse le liquide sur le premier plateau; le liquide s'écoule ainsi en sens inverse du gaz qui arrive par la partie inférieure. Il a d'ailleurs été également construit des appareils à plateaux fixes qui ont donné des résultats satisfaisants; de sorte que le mouvement de rotation des plateaux ne semble pas indispensable.

Un ÉPURATEUR LyMNn peut traiter, suivant ses dimensions, de 20 à 36.000 mètres cubes de gaz par heure.

Plusieurs installations de ce gemre sont en fonctionnement particulièrement à Barop (Angleterre) pour le chauffage des fours de l'aciérie et aux Usines de la Badische Anilin. und Sodafabrik à Ludwigshafen (Allemagne).

Gazogène Koerting (1). - La Maison Kocrting frères de Hanovre (Allemagne) a établi un type de gazogèno à lourbe représenté par la figure 3.

L appareil contporte deux zones de dislillalion, l'une supéreure sur les grilles inclinées F, l'autre inférieure sur la grille horizontale M. Il est chargé par les deux trémies A $\Lambda$ avec des briquelles de tourbe séchée a l'air qui brûle en partie sur les grilles $\mathrm{E}$, de façon à obtenir le séchage du reste du combustible el la distillation des matières volatiles contenues dans colui-ci. Les produits gazeua se dégagent par la tubulure $c$ el tue canalisation refroidic jar une enveloppe d'eau non représcntée sur la figure et par la lubu. lure $\mathrm{K}$, arrivent sous la grille $\mathrm{M}$. Ils traversent alors la masse de coke incandescendant remplissant la zone inférieure. L'humidité non condensće dans le refroidisscur est décomposée, une partie des goudrons qui ne se sont pas déposcés dans le refroidisseur esi égalenuent décomposéc el les produits gazeux encore cliargés de vésicules de goudron en suspension s'échappent par les tubulures H sous l'appel d'un ventilateur.

Pour obtenir de bons résultals avec ce gazogène, il convient de déterminer au préalable la meilleure dimension à laquelle doivent être broyées les briquettes de tourbe avanl leur chargenent dans les trémies. La durée du passage dans la zone supérieure étant, en offet, assez courte, il est néanmoins nécessaire que la tourbe en sorte complètement cokéifiée. Si la tourbe esl trop humide on en morceaux de dimensions trop grandes, elle arrivera dons la zone inférieure sans être complètement distillé ef les gaz scront beaucoul plus riches en goudrons. Fu principe, la tourbe devra ètre concassée en morccaux, d'autant plus fins que sa leneur en humidité sera plus élevée. Il conviendra également de régler l'aspiration de façon à éviter un passage direct des gaz de la zono supérieure dans les conduites d'échappement par l'étranglement $G$ dont le but est précisément, par la résistance qu'il offre, de s'opposer à ce passage.

A sa sortie du gazogène, le gaz, lorsqu'il est destiné à alimenter des moteurs ou des brûleurs à gaz est desséché el débarrassé des goudrons et des produits condensables par passage dans un scrubber à coke humide, dans un filtre à goudrons et un scrubber sec à sciure de bois. Les deux scrubbers sont du type ordinaire, nous ne les décrirons pas, mais nous insisterons sur la séparation des goudrons qui rsit d'une très grande importance.

Ainsi que nous l'avons déjà dit, une partie des goudrons s'est condensée par refroidissement à la sortie de la zone supérieure, une autre partie a été brûlée dans la zone infé-

(1) Renort on the Utilization of Peat Fuel for the nroduction of Power, Haanel, Ottawa 1912. 
rieure ; néanmoins, le gaz à sa sortie de l'appareil contient encore une certaine proportion de goudrons incondensables en suspension à l'état très divisé et qu'il est très difficile de séparer. Il est d'ailleurs impossible d'en débarrasser les gaz d'une façon absolument parfaite et en arrivant au contact des pistons à haute température, ces goudrons se déconsposent et forment des dépòts qui pourraient provoquer $\mathrm{d}$. interruptions de service si l'on n'y portait remède en temps utile. Four cela, on injecte de temps à autre dans les cylindres du moteur un mélange d'huile et d'ea'u de savon qui dissout ces dépôts. Ce procédé ne peut d'ailleurs pas être employé avec les gaz de charbon bituminenx avec lesquels il est complètement inefficace.

M. HaAnel, directeur de la Division d'essai des Combustibles au Ministère des Mines du, Canada, a réalisé un uppareil d'épuration des gaz qui débarrasse efficacement céuxci de leurs matières goudronneuses. Cel appareil (fig 4) se compose d'un cylindre métallique dont la partie inférieure est constituée par 'un scrubber à coke humide et une chambre à gaz $\mathrm{R}$. séparée de la partie supérieure par une plaque portant un orifice $O$. Sur cette plaque repose un cône A en treillis métallique donl la surfacc interne est arroséc par un jet d'eau B, tandis qu'une pomme d'arrosage $G$ ì eau chaude ou à eau froide à volonté, arrose la surface extéricure de ce cône.

Le fonctionnement de l'apparell est le suivant: Le gaz à sa sortie du générateur arrive à la partie inférieure du scrubber à coke humide qu'il traverse de bas en haut. Une partie des goudrons et des gaz condensables se dépose et est entraînée par l'eau à la partie inférieure du scrubber. Les gaz arrivent dans la chambre $R$ où ils se dilatent et se refroidissent, puis, par l'orifice $O$ passent à l'intérieur du cône en treillis métallique. Une partie des vésicules de goudrons viennent frapper contre les parois intérieures de ce cône et se réunissent en gouttes qui sont évacuées à la partie inférieure du scrubber par le jet d'eau B. Les particules de goudron qui ne sont pas encore condensées sont comprimées par le passage du gaz à travers les mailles et se déposent à la surface extérieure du cône d'où la pluie déversée par la pomme $G$ les entraîne vers le tuyau d’évacuation $C$. Lorsque la dépression dans la partie supérieure du cylindre augmente, on substitue l'eau chaude à l'eau froide, les mailles du treillis sont nettoyées et la dépression revient rapidement à sa valeur normale. Cette substitution de l'eau chaude à l'eau froide s'effectue d'ailleurs automatiquement à l'aide d'un dispositif spécial. A' la sortie de cet appareil, le gaz est suffisamment épuré et peut être utilisé directement dans les moteurs.

Gazogìnes prvers. - Il existe encore une foule d'autres gazogènes pouvant fonctionner à la tourbe. Parmi ceux-ci nous citerons :

Le Gazogène Lencauchez (1) dont il existe plusieurs types ; le gazogène Duff' à grille en dos d'âne et à prise des gaz au centre de la masse du combustible incandescent ; le gazogène Chavanne-Brun, étudié pour le fonctionnement au charbon, mais pouvant à la rigueur fonctionner à la tourbe;

Le Gazogène Pegq-Hamilton Robba $\left(^{2}\right)$, dont une installation existe aux tourbières de Maghery (Irlande) ; le Gazogène Fichet-Heurtet Sépulchre (3).

Enfin, plusieurs constructeurs étudient actuellement cette

(1) On trnnvera une bonne description de ces installations dans l'ouvrage de M. Marchis : Prnduction et utilisation des gaz pauvres.

(2) Description dans "Machines " no de Juin 1917.

"r(3) Litisine, 17 Décembre 1916. intéressante question de la distillation de la tourbe dans les gazogènes. Nous nous proposons d'ailleurs de tenir constamment nos lecteurs au courant des progrès qui se réaliseront dans cette branche de l'industrie trop longtemps négligée et destinée à apporter dans l'avenir un appoint important à la production de l'énergie.

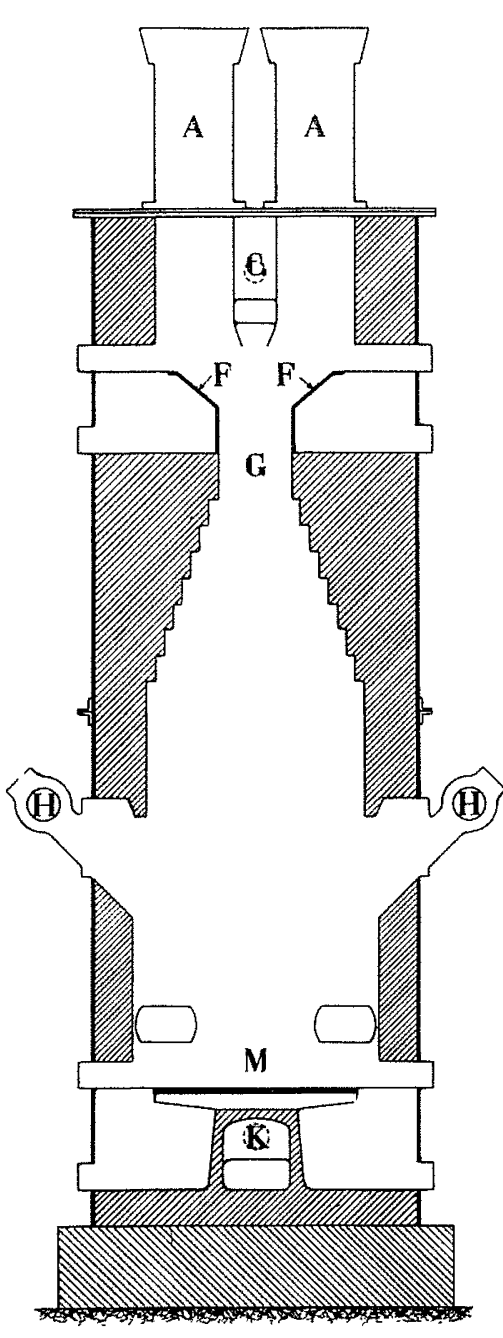

FIG 3. - GAZOGÉNE KOERTING

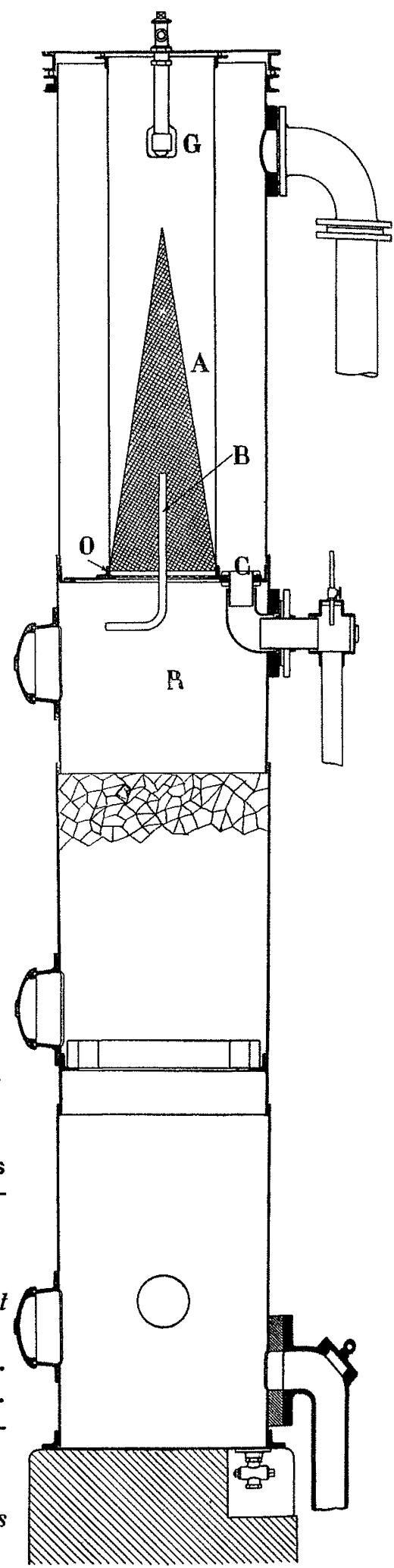

FIG. 4. - EPURATEUR A GOUDRONS ET SCRUBBER A COKE HUMIDE COMBINÉS HAANEL.

LÉGENDE DE, L'APPAREIL

$\mathrm{R}$, Chambre à gaz surmontant le scrubber.

$\mathrm{O}$, Orifice de communication.

$\mathrm{A}$, Cône en treillis métallique.

$\mathrm{B}$, Jet d'eau arrosant l'intérieur de $A$.

G, Pomme d'arrosage.

C, Canal d'évacuation des particules de goudron.

\section{LES RÉSULTATS D'EXPLOITATION}

A l'heure actuelle le meilleur résultat semble être obtenu par la Societa per l'utilizzazione dei Combustibili Italiani. L'Usine d'Orentano fut installée en 1907 et commença à fonctionner en rgro.

Celle de Codigoro entra en service en rgr2.

Dans ces deux usines on traite par le procédé Mond une 
tourbe dont la leneur en humidité est de 25 pour cent. Les $3 / 4$ de la proportion d'azote contenue dans la tourbe sont converties en sulfate d'ammoniaque dont le rendement est, pour une tourbe titrant $2.5 \%$, de $80 \mathrm{kgs}$ par lomne.

L'Usine d'Orentano traite, par jour, 5o mètres cubes de tourbe; sa production de sulfate d'ammoniaque est de jo tonnes par mois et les gaz incondensables alimenteur, en outre, une centrale à moleurs à gaz d'une puissance de Soo HP. L'usine de Codigoro tralle par jour 150 tomnes de tourbe séchée et produit ro à I 2 tonnes de sulfate d'ammoniaque. Les frais d'installation de ces deux établissements atteignent à l'heure actuelle 6.00o.ooo de francs et le prix de revient du sulfate d'ammoniaque est de $\mathbf{2} 20$ à $x 40$ francs la tonne, c'est-à-dire que, au cours actuel de plus de 300 fr. cette fabrication est très rémunératrice.

Une autre importante inslallation fonctionne depuis 1911 , à Osnabruck (Allemagne), les gaz incondensables étant utilisés pour la production de l'énergie électrique.

lette installation fonctionne d'après le procédé Mond modifié par Frank et Caro ( $\left.{ }^{1}\right)$. La tourbe humide, pouvant contenir jusqu'à 50 et même 55 pour cent d'humidité est distillée en présence d'un courant d'air chargé de vapeur d'eau à haute température. Environ 80 pour cent de l'azote contenu dans le combustible est transformé en sulfate d'ammoniaque, ce qui correspond à un rendement de $40 \mathrm{kgs}$ par tonne de tourbe sèche à $\mathrm{s}$ po'ur cent d'azote.

En outre, la distillation d'une tonne de combustible sec dégage I 250 à I 500 mètres cubes de gaz d'un pouvoir calónifique de I3oo à 1350 calories, pouvant être utilisé pour la production de l'énergie.

La centrale comprenait en I 9 I 2 trois moteurs à gaz, dont un de secours et la puissance était de $2.000 \mathrm{HP}$; le doublement était d'ailleurs prévu. L'énergie électrique produite est distribuée à la ville d'Osnabrück et dans les localités environnantes où elle est vendue à un prix inférieur à celui des autres centrales thermiques locales fonctionnant au charbon. Cette installation fut, dans le début, aux prises avec de multiples difficultés créées particulièrement par l'impossibilité de se procurer tout le combustible nécessaire, celui-ci étant en partie consommé par la population paysanne.

Une tonne de tourbe absolument sèche a produit :

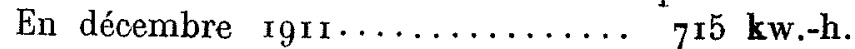

En janvier $1912 \ldots \ldots \ldots \ldots \ldots \ldots 660$ -

En février $\operatorname{rgr} 2 \ldots \ldots \ldots \ldots \ldots \ldots 675-$

Soit, en moyenne, $693 \mathrm{kw} .-\mathrm{h}$., ce qui donne une consommation de combustible sec de $1.44 \mathrm{~kg}$. par $\mathrm{kw}$.h. ou de 1.75 à r. $85 \mathrm{~kg}$. de tourbe à 25 ou 30 pour cent d'humidité.

Si l'on compare les consommations de tourbe à $25 \%$ d'humidité nécessaires pour la production de I kw.-h. par combustion et par gazéification on obtient ces chiffres

Combustion..... $2.5 \mathrm{kgs}$ de tourbe par $\mathrm{kw} . \mathrm{h}$.

Gazéification $\ldots 2.0 \mathrm{~kg}$

Pour faire une comparaison absolument exacte des deux procédés d'utilisation, il conviendrait encore de faire entrer en ligne de compte la production de sulfate d'ammoniaque réalisée par la gazéification au gazogène, et le fait que ce procédé permet l'utilisation d'un combustible ayant une teneur de 50 pour cent en humidité, ce qui permet de poursuivre la campagne d'exploitation baucoup plus tard dans l'automne.

(1) Torfmoore und Kraftuebertragung. - Elektrotechnisch Zeits chrift 7 Mars et 4 Avril 1907.

Mour kultur und Torivewertung. - N. Garo. - Elektrotechn. Zeils 10 Novembre 1910
Dans une comparaison impartiale, il y aurait encore lieu de tenir compte de la nature des appareils d'utilisation, turbines à vapeur ou moteurs à gaz, l'inconvénient de ceux-ci étant leur faible capacilé de surcharge.

\section{AUTRES UTILISATIONS DE LA TOURBE}

La lourbe, mélangée à du goudron, peul constituer des briquettes convenant hes blen au chaulfage domestique. Une fabrication de ce gemre avail été entreprise à Rocheforl; elle dût être interrompue par suite du manque de lourbe.

L'usine à gaz de la mòme ville emplore dans ses cornues de distillation un mélange de tourbe el de houille qui donne, paraît-il, dexcellents résultats.

Des essais dans ce sens ont égalemenl été elfectués à l'Usine à Gaz de berne (Suisse). Les essais ont porté sur 36 stères de tourbe provenanl de Moosseedorf' (près de Berne), extraite à la machine et contenant 33 pour cent d'humidilé. La dishllation a élé effecluée en ia heures dans des fours verticaux Dessau et des fours inclinés Doze. Les résultats suivants ont été oblenus :

Composition du GAZ :

Acide carbon. 25.8 p. 100

Carb. lourds. $3.3-$

Oxyde de carb. $15.7 \quad$ p. 100 Ilydrogène . 4 41.9 Méthane ... $10.5 \quad-$ Oxygène ... $0.00-\quad$ Azote ..... 2.8 -.

Le coke de lourbe est difficile à éleindre; le goudron résultant de cette distillation a une couleur brunatre of répand une odeur de laine brùlée.

Cependant, des essais ont eu lieu dernièrement au Laboratoire de Recherches de l'Usine à Gaz de la Villette à Paris, essais dont la conclusion a été que l'emploi de la tourbe dans les cornues de distillation sèche ne pouvait être envisagé favorablement par suite de la trop forte teneur en acide carbonique des gaz produits et de la mauvaise qualité du coke abtenu, dont l'extinction doil avoir lieu à l'éloulfoir, l'eau le transformant en boue ei la quantité étant insuffisante même pour assurer le chaulfage des cornues.

La tourbe mousseuse ou lourbe de surface trouve son principal emploi dans la constitution des litières pour le bétail en verlu de son grand pouvoir absorbant pour l'humidité et l'ammoniaque. Ces qualités jointes à ses propriétés antiseptiques en répandent également l'emploi en médecine et, comme on le sait, porr la confection des sousvètements hygiéniques.

De nombreux essais ont été galement tentés pour substituer la tourbe à la pâte de bois dans la fabrication du papier. Ce mode d'emploi ne paraît pas avoir donné des résultats entièrement satisfaisants.

Enfin, la tourbe est également utilisée pour la constitution de nitrières destinées à nitrificr l'azote ammoniacal, apporté sous forme de sulfate d'ammoniaque.

Pour cette question intéressante, mais qui sort du cadre de cette Revue, nous renverrons le lecteur aux travaux de Boussingault et Schlesing, ainsi qu'à l'ouvrage de M. J. Escard : La fabrication électrochimique de l'acide nitrique et des composés nitrés à l'aide des éléments de l'air.

Pierre Guieu,

Ingénieur, Ancien Elève de l'Ecole de Physique et de Chimie de Paris.

\section{LA SOUFFLANTE DANS LES COKERIES}

La question de la circulation des gaz dans les installations qui utilisent la Tourbe par distillation ayant un lien aveć l'étude qui précède, nous jugeons a propos de placer ici cet extrait de: "Revue De B. B. C. No 4 (Avril-Septembre 1917), p. 75-79 ". 
L'utilisation rationnelle de la tourbe par distillation pyrogénée avec récuperation des sous-produits contenus dans les gaz, prend actuellenient une grande importance.

Les produits de celle distillation sont : le coke, combustible à pouvor caloritique élevé, le gaz utilısé pour le chauftage ou l'éclairage et les sous-produits, parmi lesquels particulièrement les goudrons, lo benzol el l'ammoniaque.

La carculation du gaz dans linstallabon est assurée par des soufflantes spécrales diles extracteurs de gaz, donl l'étude constitue l'objet princupal du présent article. L'extraction des goudrons s'opère aisément, soit dans les conduites, soit dans des appareils spéciaux, les séparateurs de goudrons, dont le fonctionnement est bien connu.

La récupération de l'ammonaque sous forme de sulfate acide poul avoir lieu par trois procédés différents : Le procédé direct, dans lequel l'acide sulfurique agit sur les gaz, le procédé indirech, dans lequel l'acıde agit sur les eaux ammoniacales résultant du lavage des gat, le procédé semi-indirect, combinaison des deux précédents. La descriplion de ces procédés est accompagnée de schémas d'installation et de diagrammes de température des gaz aux divers points de leur trajet.

L'emplacement de l'extracteur à gaz varie suivant le procédé. Dans le procédé indirect, il est traversé par des gaz dont la température est de $35^{\circ} \mathrm{C}$, landis que, dans le procédé direct, cetle lempérature monte à $60^{\circ} \mathrm{C}$, les gaz sont, par conséquent, beaucoup plus riches en goudrons qui se déposent, pendant la marche, sur le rotor de la soufflante, d'où la force contrifuge les projclle sur les parois ; pendant les arrêts, ils pénètrent par les joints qui ne sont jamais parfaitement herméliques el encrassent le rotor. Il peut en résulter une répartition inégale des masses de celui-ci, provoquant des vibrations dans la machine.

II est donc nécessaire, pour obtenir un bon fonctionnement, de réduire au minimum l'encrassement de l'extracteur à gaz par les goudrons. Dans ce but, l'auteur recommande de disposer un séparateur dio goudrons immédiatement, avant l'entrée des gaz dans l'appareil.

Néanmoins, il est impossible d'éviter, d'une façon absolue, I'intraduction de goudrons dans l'extracteur à gaz. Il convient de prendre des dispositions pour en assurer l'évacuation : En marche, les goutelettes de goudron projetées ainsi que nous l'avons dit, contre les parois se rassemblent à la partie inférieure où il suffira de disposer une conduite de purge plongeant, afin d'éviter l'échappement des gaz, dans un pot de syphonage, à une profondeur suffisante pour que la pression de J'eau soit supérieure à la pression des gaz. De plus, le pot de syphonage devra être placé assez bas pour que des rentrées d'eau ne puissent se produire à la mise en marche. Après un arrêt prolongé, l'extracteur est purgé à la vapeur, dont la chaleur liquéfie les goudrons qui se rassemblent à la partie inférieure. - Sous l'action de l'acide sulfurique, le goudron se transforme en résine qui, se fixant au rolor, devient très difficile à extraire. C'est là un second inconvénient du procédé direct pour la récupération "de l'ammoniaque.

Enfin, un exposć des conditions générales de l'installation d'une cokerie nécessaires au hon fonctionnement de l'extracteur à gaz $\because$ nine cette étude. P. G.

\section{UN PROGRAMME DAMÉNAGEMENT DU RHONE}

La grande question de l'Aménagement du Rhône a déjà fait l'objet de nombreuses études dans notre journal. Le courant se dessine de plus en plus en faveur d'un programme d'ensemble et d'une concession globale. Notre prochain numéro donnera,comme base de discussion le texte complet d'un projet de constitution d'une grande Compagnie Nationale du Rhône (forces motrices, navigation, exploitation du canal, aménagement des terrains, têtes d'eau d'irrigations), qui devrait grouper toutes les collectivilés intéressées, projet que son auteur M. J. MatTre soumet sujourd'hui à l'Administration supérieure en même temps qu'aux Shambres de commerce el aux Conseils généraux de la région.

\section{COUMISSION EXTRAPARLEMENTAIRE DES FORCE'S HYDRAULIUULS}

\section{Texues des Proces-Verbaux des Séances}

\author{
SÉANCE DU 14 JUIN 1917 (10 Séance)
}

Presenls : MiM. hlotz, Président ; Alexandre Bérard, Lourties, Léon Perher, Miargaine, M. Kéville, Berthélemy, Blazeix, Cimarmeil, Manied, Salles, Ciolonel Maison, Commandant Cianen, Uabat, 'I moté, Dupontell, du Longbois, Rousseau, Tisserand, bouchayer, Bougault, Cordier, Marlo, Pinot, 'I eissier, Layaud, Perier du féral, Petit, Lortie, Conte.- Excusé: M. Monestier.

La séance est ouverte à ro h. I/4.

M. Le Président. - Nous avons fait tirer et on vous a distribué le lexte de toules les dispositions adoptées jusqu'à ce jour. Le rapprochement de ces décisions fait ressorlir la nécessité d'une correction que je signale : Dans le $5^{\circ}$ du nouveau texte, les mots " assimulés à ides travaux publics" semblent constituer une contradiction avec ce qui est dit plus loin, au $\S$ I 4 commençant ainsi "Assimulable aux concessions de mines, etc... " 11 y aurait lieu de supprimer, dans le panagraphe 5, les mots : "assimilés à des travaux pubhes " pour qu'on ne puisse pas nous faire le reproche de cetle apparente contradiction.

Ensuite, il convient d'ajouter deux mots oubliés dans le $\S 16$, où il taut lire : " ... produit de la hauteur de chute... ")

Il m'a été dıt que MM. les industrıels ne pourraient pas assister à nos réumıons pendant la quatrième semanne de juin. Nous pourrions suspiendre nos séances pendant cette semaine, et ce délai serait utilisé pour rédiger le texte des articles du projet de loi correspondant à toutes les décisions aujourd'hui acquises. Il conviendrait que nos rédacteurs fussent désignés, dès maintenant, pour pouvoir y réfléchir. Je vous proposerais de choisir MM. Rousseau, Conseiller d'Etal ; M. Berthélemy, Professeur à la Faculté de Droit et M. Petit, Inspecteur des Finances, qui consulteraient naturellement les admunistrations intéressées et les représentants de l'industrie, ainsi que M. Margaine, auteur d'une proposition de loi.

Nous continuons maintenant notre examen. Il s'agit d'établir la limite entre le décret et la loi. Qui demande la parole sur la fixation de cette limite?

M. Bentí́lemy. - Il y a accord entre tout le monde. Je crois que tous les projets ont propasé de s'en référer au décret de $187^{\circ}$.

M. Mamev. - Il faudrait peut-être s'inspirer des chiffres qui ont été votés par le Parlement dans l'article I 5 de la loi de finances du 28 septembre r 916 , en tenant compte de ce fait que nous avons changé d'unité. Cette loi indiquait les chiffres suivants : 20 kilomètres mesurés suivant le lit et 15.000 kilowatts à l'étiage. Ces r 5.000 kilowatts mesurés à l'étiage doivent équivaloir à environ 30.000 kilowatts aménagés. Nous pourrions donc admettre : 20 kilomètres de longueur mesurés suivant la dérivation, et 30.000 kilowatts aménagés pour rester logiques avec nous-mêmes.

M. Cordibr. - Il me semble qu'il est intéressant, étant donné le soin qu'elle apporte à rédiger la nowvelle loi, de ne faire intervenir le Parlement quie dans les cas exceptionnels : Une usine de 30.000 kilowatts, c'est une usine actuollement moyenne. Si l'on s'en réfène au projet d'aménagement die la Durance, on verra que, si on prend le débit moyen, presque toutes les usines projetées dépassent la puissance de 30.000 kilowatts. Par conséquent, si on fixe le chiffre de 30.000 kilowatts comme limite, je crois qu'on fera une loi qui s'appliquera rarement étant donné les progrès de l'industric. Il y a dix ans, on considérait une usine de ro.000 ou 15.000 kilowatts comme une usine puissante; dans dix ans, on pourra considérer une usine de 50.000 kilowatts comme une usinc normale ; par consóquent, on arriverait à ce résultat que, dans quelques années, toutes les chutes devraient faire l'objet d'une loi, ce qui semble le contraire de ce que nous voulons. 